Carr, Kristen

[32]

\section{Relationship between gene expression profile and clinical outcome in malignant melanoma}

\author{
Kristen Carr ${ }^{1,2}$, Mike Bittner², Yidong Chen², Paul Meltzer², \\ Paul Duray ${ }^{3}$, Vern Sondak ${ }^{4} \&$ Jeffrey Trent $^{2}$ \\ ${ }^{1}$ Howard Hughes Medical Institute and Cancer Genetics Branch, National \\ Human Genome Research Institute, National Institutes of Health, Bethesda, \\ Maryland, USA \\ ${ }^{2}$ Cancer Genetics Branch, National Human Genome Research Institute, National \\ Institutes of Health, Bethesda, Maryland, USA \\ ${ }^{3}$ Surgery Branch, National Cancer Institute, National Institutes of Health, \\ Bethesda, Maryland, USA \\ ${ }^{4}$ Department of Surgery, University of Michigan, Ann Arbor, Michigan, USA
}

Cutaneous melanoma is the most serious form of skin cancer and one of the most common cancers in young adults. Its incidence is increasing at a significant rate, and the long-term survival rate for patients with melanoma has not improved markedly since the 1970s. Our laboratory identified unexpected subtypes of human cutaneous melanoma and observed a unique pattern of gene expression in highly invasive melanomas ${ }^{1}$. However, the study design for this initial report did not allow a direct correlation of gene expression profiles with disease progression or response to therapy. We have begun to address the relationship between gene expression profile and clinical outcome by collecting and analyzing a set of melanoma tumor biopsy samples with known clinical outcome. We have supplemented this set of samples with a melanoma tissue microarray. The examination of gene expression patterns of melanoma tumors will provide a unique opportunity to study a homogeneous group of patients and determine whether gene expression patterns can assist in predicting disease progression or therapeutic response.

1. Carr, K. et al. Nature 406, 535 (2000).

Chen, Danian

\section{Genomic amplification in retinoblastoma narrowed to $1.2 \mathrm{Mb}$ on chromosome $6 \mathrm{p}$ containing a novel kinesin-like gene, RBKIN}

\author{
Danian Chen ${ }^{1,3}$, Jeremy Squire ${ }^{1,2} \&$ Brenda Gallie ${ }^{1,2}$ \\ ${ }^{1}$ Division of Cancer Informatics and Division of Cellular and Molecular Biology, \\ Ontario Cancer Institute/Princess Margaret Hospital, University Health \\ Network, 610 University Avenue, Toronto, Canada ${ }^{2}$ Departments of \\ Ophthalmology and Laboratory Medicine and Pathobiology, University of \\ Toronto, Canada ${ }^{3}$ Department of Ophthalmology, West China University of \\ Medical Sciences, Chengdu 610041, The People's Republic of China
}

Retinoblastoma is a rare tumor that arise in human retina when both RB gene alleles are mutated. All retinoblastoma have additional genomic changes evident on karyotype and comparative genomic hybridization. We have previously shown a high frequency of the $i(6 p)$ marker chromosome and gain of 6 p22 in retinoblastoma. We now report a minimal region of $6 \mathrm{p} 22$ gain in retinoblastoma that spans 1.2 $\mathrm{Mb}$ detected by quantitative multiplex PCR. Using public databases, we determined that the sequence of this region was contained in three contigs. The STS marker with the highest frequency of genomic gain was contained within one fully sequenced PAC AL023807. Exon prediction indicates three unigene clusters within this PAC, one with 19 cDNA sequences in the dbEST. By analysis of those sequences we predicted a partial cDNA sequence, which has homology to the motor domain of the kinesin gene family. We generated several nested PCR primer pairs for rapid amplification of cDNA ends (RACE) and obtained from a retinal cDNA library a previously uncloned kinesin family gene, RBKIN, expressed in retina and included in the region of genomic gain in retinoblastoma. RBKIN is $5,850 \mathrm{bp}$ and is homologous with mouse Kif13a. It is expressed in all adult human tissues tested including retina, as well as retinoblastoma, but its expresion level is very low in most fetal tissues. RBKIN and/or another gene in the same $1.2 \mathrm{Mb}$ of 6 p22 are likely to be oncogenes that contribute to initiation and malignant progression of retinoblastoma.

Chen, Sei-Yu

$[34]$

\section{Genomics-based strategies for the identification of lung cancer diagnostic targets}

Sei-Yu Chen, Muriel Kmet, Carrie Drumright, Brandon Liang, Vu Viet Hoang, Herve Recipon, Yongming Sun, Ping Hu \& Roberto A. Macina

diaDexus, Inc., 3303 Octavius Drive, Santa Clara, California 95054, USA

Lung cancer is the most common cancer in men and women. In 2000 it caused an estimated 156,000 deaths, accounting for $28 \%$ of all cancer-related deaths. We have screened for differentially expressed genes in lung cancer using complementary DNA database mining and suppression subtractive hybridization based on the polymerase chain reaction. These genomics methods have been used to identify lung cancer genes that are tissue-specific, lung cancer-specific or both. We generated eight subtracted libraries using different subtraction combinations and sequenced 10,000 clones from each library. To confirm the differential expression of the candidate genes obtained, we used the real-time polymerase chain reaction with Taqman fluorescent probe assays. We generated gene expression profiles for some of these genes on different anatomical sites and in different disease states. We used this validation strategy to analyze 46 potential candidates. The RNA expression for 7 of these 46 genes was tested in more than 120 tissue samples. All genes showed high tissue-specific expression and overexpression in $45-67 \%$ of the lung cancer samples compared with the expression in normal adjacent tissues from the same individual. Full-length cloning, gene expression in bacteria and antibody production for these genes are under way. Once all the reagents are obtained, we will develop immunoassays to evaluate their utility as biomarkers for lung cancer. These genes, either individually or grouped in panels, could be of potential use as new tumor markers for early detection, differential diagnosis, prognosis, disease monitoring or cancer surveillance. They could also be useful as new therapeutic targets.

Chen, Yidong

[35]

\section{cDNA microarray analysis using both gene expression ratios and intensities}

Yidong Chen ${ }^{1}$, Hongen Zhang ${ }^{1}$, Sujatha Panavally ${ }^{1}$, Edward Dougherty ${ }^{2}$, Michael Bittner ${ }^{1}$, Paul Meltzer ${ }^{1} \&$ Jeffery Trent ${ }^{1}$

${ }^{1}$ Cancer Genetics Branch, National Human Genome Research Institute, National Institutes of Health, Bethesda, Maryland, USA

${ }^{2}$ Department of Electrical Engineering, Texas A\&M University, College Station, Texas, USA

In typical complementary DNA microarray experiments, two fluorescently labeled RNAs are hybridized to an array of cDNA probes on a glass slide, and their rela- 
Abstracts: Session I

tive gene expression intensities, ratios or both are measured in order to quantify the gene expression level relative to its reference sample. Our early reports discussed a systematic data extraction algorithm in which a unique method of extracting gene expression intensities and ratios along with an adaptive ratio confidence interval, measurement qualities of gene expression ratios and intensities were presented. In many methods of gene expression data analysis, only expression ratios or normalized intensities are employed because of insufficient assessment at the individual data points (clones). Common practice dictates that data derived with poor measurement quality-such as expression ratios derived from weak reference expression levels or noise-corrupted measurements—shall not be used in the analysis. We present an automatic decision-making process for various algorithms, such as gene expression clustering and classification, in which gene expression ratios and intensities are chosen to participate in the analysis according to their measurement quality, expression signal-to-noise ratio relative to the reference channel and other parameters derived from cDNA microarray image analysis software.

Chen, Zhong [36]

\section{Molecular profiling of metastatic tumor progression of a murine squamous cell carcinoma by differential display and CDNA microarray reveals dysregulated expression of genes related to the nuclear factor- $k b$ signal pathw ay}

\author{
Gang Dong ${ }^{1}$, Elena Loukinova ${ }^{1}$, Zhong Chen ${ }^{1}$, Lisa Gangi ${ }^{2}$ \\ Edison Liu² \& Carter Van Waes ${ }^{1}$ \\ ${ }^{1}$ National Institutes of Health, Bethesda, Maryland, USA National Cancer \\ Institute, National Institutes of Health, Bethesda, Maryland, USA
}

To identify changes in gene expression associated with tumor progression and metastasis in vivo, we investigated differential gene expression in a metastatic squamous carcinoma model established in syngeneic mice, including a tumorigenic line PAM 212, and metastatic sublines derived from PAM 212 tumors, using mRNA differential display (DD) and cDNA microarrays. Using DD, seventy-two candidate cDNAs were detected, and thirty-four cDNAs were confirmed to be differentially expressed by northern blotting analysis. Global mRNA expression profiles were generated using an NCI mouse Oncochip composed of four thousand elements representing known genes and ESTs, plus 57 of the candidate cDNAs detected by DD to facilitate data validation. Clustering analysis of array results from metastatic cell lines and tumors identified a subset of genes that exhibited increased expression in the metastases, revealed that 22 unique clones are highly homologous to previously identified genes, and nine novel cDNAs. Strikingly, $10 / 22$ of the genes identified have been associated with activation of the Nuclear Factor-kB signal transduction pathway. One of the genes identified, Gro-1, was recently confirmed to promote tumor growth, metastasis and angiogenesis of SCC in vivo in a separate report. These results demonstrate that early response pathway components and down stream genes related to NF- $\mathrm{KB}$ are expressed with metastatic tumor progression. Functional genomic approaches may promote a better understanding of the repertoire of related genes and molecular pathways involved in tumor progression and metastasis.
Chodosh, Lewis

[37]

\section{Functional analysis of mammary development using oligonucleotide microarrays}

Stephen Master, Jennifer Hartman, Alexander Stoddard, Elizabeth Keiper, Susan Moody, Celina D'Cruz $\&$ Lewis Chodosh

University of Pennsylvania School of Medicine, Philadelphia, Pennsylvania, USA

Epidemiological and animal studies clearly demonstrate that reproductive events such as puberty, pregnancy and parity play a critical role in the determination of breast cancer risk. We have carried out messenger RNA expression profiling using high-density synthetic oligonucleotide microarrays to identify genes that are differentially expressed between various developmental stages of the murine mammary gland. The use of DNA microarrays to study vertebrate development presents unique analytical challenges compared with expression profiling of homogeneous cell populations. These challenges include accounting for the impact of complex changes in the abundance of multiple cell types on gene expression profiles, as well as identifying functionally relevant patterns of gene expression in the absence of detailed prior knowledge either of the developmental system or of the genes expressed. In order to address these challenges, we have developed a general approach that permits the unbiased identification of biologically relevant patterns of gene expression by identifying statistically significant associations between clustered gene expression patterns and functional gene categories. We have tested the applicability of this approach by analyzing the expression of approximately 5,500 genes during 13 stages of murine mammary gland development. Our findings confirm the utility of this method by demonstrating the ready identification of cellular processes and pathways of known importance in mammary development, as well as shifts in the relative abundance of different cell types within the gland. This approach permitted the identification of genetic pathways with previously unsuspected patterns of developmental regulation, including those involved in fatty acid metabolism, angiogenesis and extracellular matrix synthesis. Our results demonstrate the ability of this analytical approach to suggest new hypotheses regarding mammary development and indicate that this approach will be broadly applicable to the study of complex tissues.

Chuang, Y. Eric

$[38]$

\section{Effects of p53 in human lymphoblast cells following ionizing radiation using $7 \mathrm{~K}$ cDNA microarrays}

Y. Eric Chuang ${ }^{1}$, Howard Liber ${ }^{2}$, Louis Staudt ${ }^{1}$

$\&$ James Mitchell ${ }^{1}$

${ }^{1}$ Division of Clinical Sciences, National Cancer Institute, National Institutes of Health, Bethesda, Maryland, USA

${ }^{2}$ Department of Radiation Oncology, Massachusetts General Hospital, Boston, Massachusetts, USA

Genomic instability is a characteristic of many human cancers. It has been well documented, utilizing endpoints such as karyotypic instability and gene amplification, that alterations in the tumor suppressor p53 are related to genomic instability at the chromosomal level. The p53 protein has been implicated in multiple cellular responses related to DNA damage, including apoptosis, cell cycle control and DNA replication, repair and transcription. Alterations in any of these processes could be related to increased genomic instability. We previously compared radiation-induced mutagenicity among human B lymphoblast cell lines with dif- 